\title{
Sobre LA PRESENCIA de MEGALASTRUM SUBStRIGOSUM (Dryopteridaceae) en Argentina
}

\author{
GONZALO MARQUEZ1 ${ }^{1}$ AGUSTINA YÁÑEZ² y SANTIAGO KELLY1
}

\begin{abstract}
Summary: About the presence of Megalastrum substrigosum (Dryopteridaceae) in Argentina. This paper presents the first record of Megalastrum substrigosum from Argentina, in Department San Pedro, Misiones province. We present a description of the species, a distribution map, and illustrations of the diagnostic characters. A dichotomous key is included to differentiate the three Megalastrum species from Misiones.
\end{abstract}

Key words: Megalastrum substrigosum, Dryopteridaceae, Misiones, Argentina.

Resumen: En este trabajo se cita por primera vez a Megalastrum substrigosum para Argentina, en el departamento de San Pedro, provincia de Misiones. Se presenta una descripción general de la especie, el mapa de su distribución en el país e ilustraciones sobre los caracteres más importantes para su determinación. Se incluye una clave dicotómica para diferenciar las tres especies presentes en Misiones.

Palabras clave: Megalastrum substrigosum, Dryopteridaceae, Misiones, Argentina.

\section{INTRODUCCIÓN}

El género Melagastrum Holttum (Dryopteridaceae) cuenta con 91 especies, que se distribuyen principalmente en la región Neotropical, desde México y Cuba, hasta el sur de Chile. Solo tres especies crecen en el Viejo Mundo (Moran et al., 2009; 2014a, 2014b). Se caracterizan por ser plantas terrestres que habitan el sotobosque de selvas o bosques tropicales y subtropicales.

Megalastrum se diferencia de otros géneros de la familia Dryopteridaceae por el grado de división de la lámina, la venación y el tipo de pelos en la cara adaxial de los ejes. Las pínulas basiscópicas basales se convierten poco a poco en decurrentes y ampliamente adnatas al raquis de la pinna. Así mismo, la vena que alimenta al segmento adnato surge de la costa, no de la cóstula. Esto es único entre los helechos dryopteroides.

1 Cátedra de Palinología, Facultad de Ciencias Naturales y Museo, UNLP, Paseo del Bosque s/n, B1900FWA, La Plata, Argentina. E-mail: cosme@fcnym.unlp.edu.ar

2 Museo Argentino de Ciencias Naturales Bernardino Rivadavia, CONICET, Av. Angel Gallardo 470, CABA, Argentina.
$\mathrm{Si}$ bien se pensaba que este género estaba estrechamente relacionado con Ctenitis (C. Chr.) C. Chr., recientes estudios filogenéticos basados en ADN revelan que Rumohra Raddi es el género hermano de Megalastrum, y que estos últimos dos géneros están emparentados con Lastreopsis Ching (Schuettpelz \& Pryer, 2007; Labiak et al. 2014).

En Argentina se conocían hasta el momento cinco especies: tres en el NOA (M. adenopteris (C. Chr.) A.R. Sm. \& R.C. Moran; M. pulverulentum (Poir.) A.R. Sm. $\&$ En Argentina se conocían hasta el momento cinco especies: dos en el NOA (M. adenopteris (C. Chr.) A.R. Sm. \& R.C. Moran; M. pulverulentum (Poir.) A.R. Sm. \& R.C. Moran), una en el sur del país (M. spectabile (Kaulf.) A.R. Sm. \& R.C. Moran var. spectabile) y dos en el NEA M. connexum (Kaulf.) A.R. Sm. \& R.C. Moran; M. crenulans (Fée) A.R. Sm. \& R.C. Moran) (Ponce, 2001; Marquez et al., 2006; Salino \& Ponce, 2008; Tressens et al., 2008, Sundue et al. 2010).

En este trabajo se amplía la distribución de Megalastrum substrigosum R. C. Moran, J. Prado $\&$ Labiak y se da a conocer su primer registro para la Argentina. También se describe e ilustra a este taxón y se propone una clave para diferenciarla de 
las otras especies del mismo género en la provincia de Misiones.

\section{Material y Método}

En este trabajo se utilizaron ejemplares coleccionados en el Departamento de San Pedro, Misiones, Argentina, en un viaje de campaña realizado durante el mes de mayo de 2014. Los mismos fueron depositados en el herbario LP.

El material fue herborizado por la técnica habitual, donde se utilizaron hojas de papel de diario y alcohol $96^{\circ}$, con el fin de ayudar en la deshidratación.

En la identificación de los ejemplares se utilizó la flora de Santa Catarina (Sehnem, 1979) y del Cono Sur (Salino \& Ponce, 2008) y los trabajos de Marquez et al. (2006) y Moran et al. (2009).

\section{Resultados}

Megalastrum substrigosum R. C. Moran, J. Prado \& Labiak, Amer. Fern Jour. 99 (1): 23-39. 2009. Tipo. Brasil, Espírito Santo, Mun. Castelo, Parque Estadual do Forno Grande, afloramentos rochosos, com matas úmidas nos vales, $20^{\circ} 19^{\prime} 16^{\prime} \mathrm{S}, 41^{\circ} 59^{\prime} 50^{\prime \prime} \mathrm{W}, 1300$ m, 18 Jul 2007, Labiak et al. 4222 (holotipo: UPCB; isotipo: NY01010306!; NY01010307!). (Fig. 1, Fig. 2)

Plantas terrestres. Rizomas erectos. Frondes de aproximadamente $1,5 \mathrm{~m}$ de largo. Bases de los pecíolos con escamas de hasta $2 \times 0,1 \mathrm{~cm}$, lineares, denticuladas, amarillas a castañas. Laminas $1 \mathrm{~m}$, 2-pinnado-pinnatifidas. Pinnas basales de $55 \mathrm{~cm}$, inequilaterales, peciólulos de $2 \mathrm{~cm}$. Ráquis pubescentes en la cara abaxial, con pelos subestrigosos de hasta 0,5 $\mathrm{mm}$, escamas de hasta $3 \mathrm{~mm}$, lanceoladas, marrones, denticuladas; la cara adaxial densamente pubescente, pelos subestrigosos de 0,5 $\mathrm{mm}$. Cóstulas abaxialmente pubescentes y escamosas, con escamas rojizas; abaxialmente pubescentes, sin escamas. Venas visibles en ambas superficies, pubescentes en el parte abaxial y con pelos subestrigosos en la parte adaxial; margen de la lámina ciliado, con pelos subestrigosos de hasta 0,2 $\mathrm{mm}$. Soros redondeados e indusio ausente. Esporas monoletes, crestadas.

Distribución y hábitat. Si bien fue descrita por Moran et al. (2009) como endémica del estado de Espíritu Santo, Brasil, en este trabajo se cita por primera vez para el Departamento de San Pedro en la provincia argentina de Misiones.

Observación. M. substrigosum es la sexta especie de Megalastrum presente en Argentina y la tercera encontrada en el NEA. Esta especie se reconoce por los pelos subestrigosos en las superficies abaxiales de los raquis, costas, cóstulas y venas; también se observan finos pelos cortos en las porciones de láminas entre venas, en la cara abaxial. Crece dentro de selvas subtropicales y forma parte del estrato herbáceo.

Material examinado. ARGENTINA. Prov. Misiones: Dpto. San Pedro, Parque Provincial Moconá, 20-V-2014, Kelly et al. 3 (LP); Ídem, Reserva Esmeralda, 23-V-2014, Kelly et al. 48 (BA, LP).

\section{Clave para diferenciar a las especies argentinas del género Megalastrum}

1 Indusio presente. 2

2. Indusio que cubre completamente el soro, persistente. M. crenulans (Misiones)

$2^{\prime}$. Indusio pequeño, fugaz. M. adenopteris (Jujuy y Salta)

1 '.Indusio ausente. 3

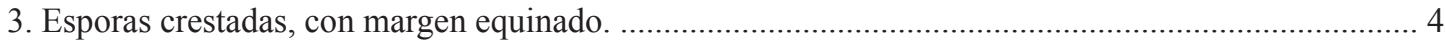

$3 '$.Esporas equinuladas. M. substrigosum (Misiones)

4. Cara abaxial del raquis pubescente, con pelos subestrigosos.

4'. Esporas crestadas. Cara abaxial del raquis glabra o glabrescente, los pelos nunca subestrigosos. ... M. connexum (Corrientes y Misiones)

5. Pinna basal 4-pinnado-pinnatisectas, lámina entre las venas densamente pubescente, crece en el Noroeste del país. M. pulverulentum (Jujuy, Salta y Tucumán)

5'. Pinna basal 3-pinnado-pinnatisectas, lámina entre las venas glabras o escasamente pubescente, crece en la selva Valdiviana en el sur del país. ..... M. spectabile (Chubut, Neuquén y Río Negro) 


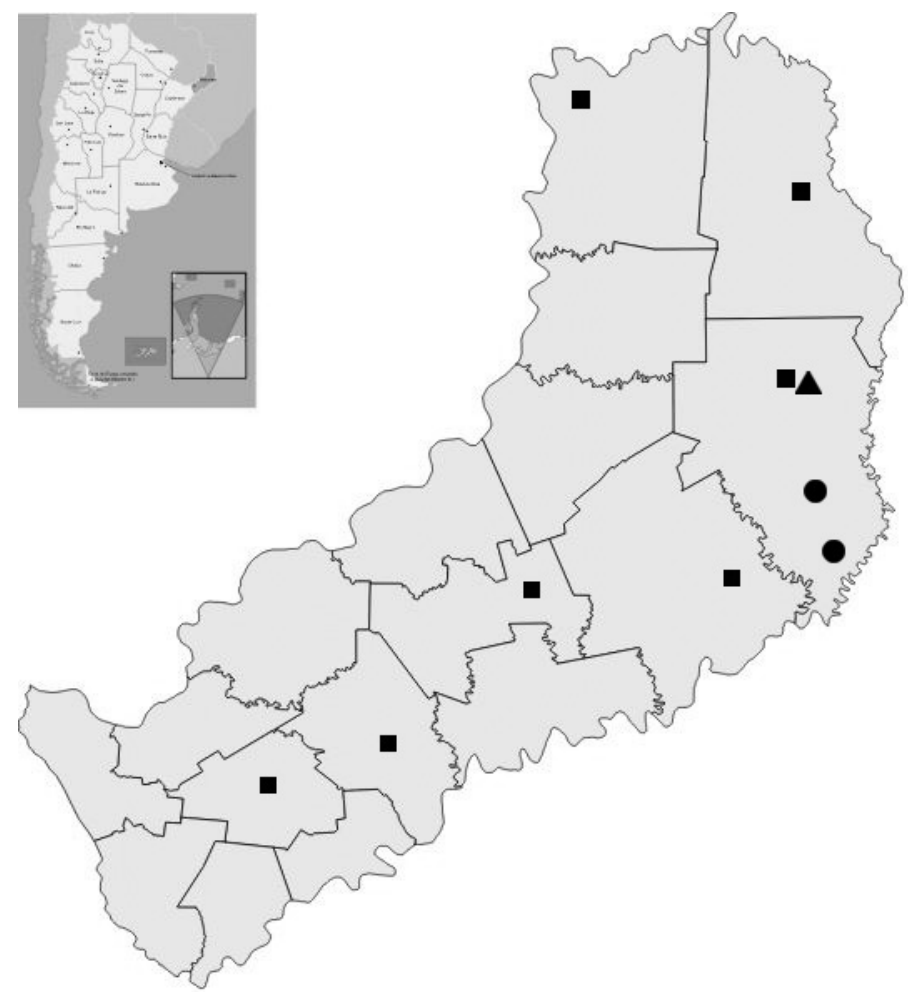

Fig. 1. Distribución de las especies de Megalastrum en Misiones: $M$. connexum (๘), M. crenulans ( $\mathbf{\Delta})$ y $M$. substrigosum $(\bullet)$.
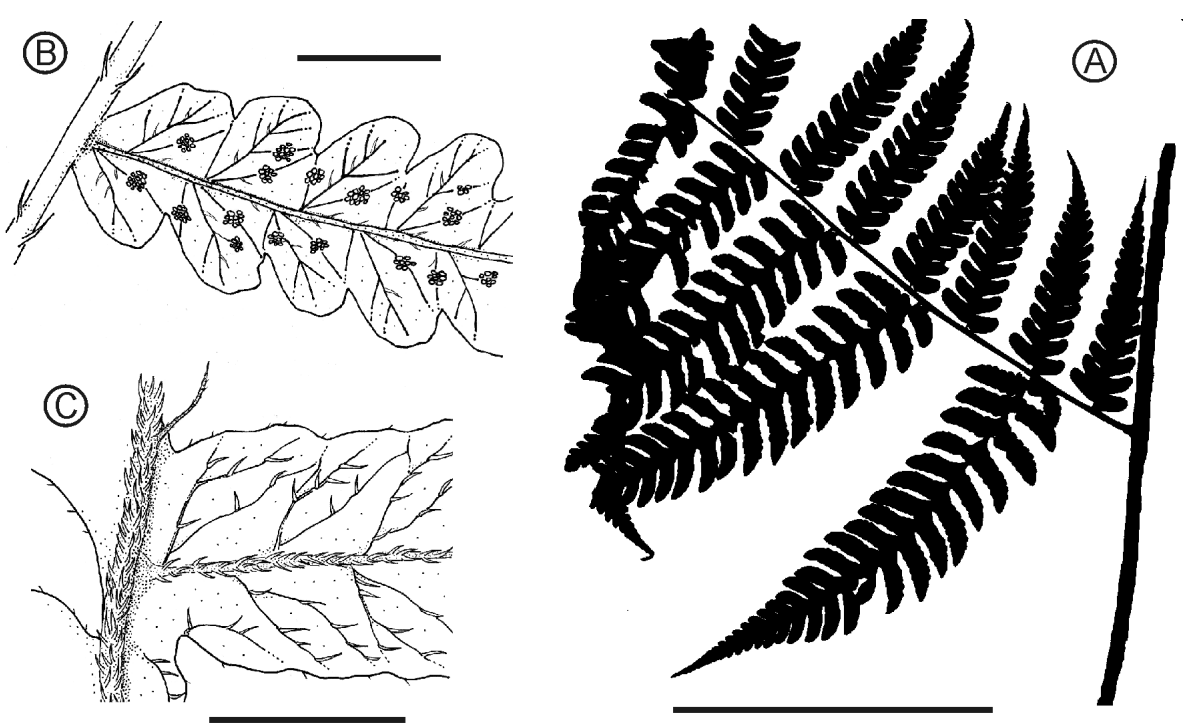

Fig. 2. A: Aspecto general de la pinna basal, donde se observan las pínnulas basiscópicas diferenciadas de las acroscópicas. Barra: $10 \mathrm{~cm}$. B: Aspecto de la cara abaxial de una pinnula. Se observan los soros desnudos y pelos subestrigosos en la cóstula y venas laterales. Barra: 0,5 cm. C: Cara adaxial de una pinnula. Costa y cóstulas densamente pubescentes. Pelos subestrigosos. Barra: $0,5 \mathrm{~cm}$. 


\section{Discusión y Conclusiones}

El género Megalastrum está pobremente estudiado en la Argentina en general, y en Misiones en particular. Megalastrum connexum es una especie muy abundante en esta última provincia y su diferenciación con el resto de los helechos es bastante sencilla. Se distingue de Megalastrum crenulans, la otra especie del género presente en el área, por presentar soros desnudos. No obstante, es posible que los especialistas no se hayan detenido a coleccionar una mayor cantidad de ejemplares exindusiados de este género, asumiendo que se trataba en todos los casos de M. connexum.

A partir de la publicación de la revisión de Megalastrum para Brasil (Moran et al. 2009) llamó la atención a los autores del presente trabajo la gran diversidad de especies del género en el sur de ese país, por lo cual se comenzó a indagar sobre el grupo en el Noreste de Argentina y se colectaron los ejemplares estudiados en este trabajo. Cabe destacar que sólo fue posible llegar a una determinación certera de los ejemplares de $M$. subestrigosum una vez que los mismos fueron observados en detalle a la lupa y se identificaron los pelos subestrigosos característicos que diferencian a la especie de $M$. conexum.

De esta manera, queda evidenciado el valor de la identificación de microcaracteres de importancia sistemática, no solo en la determinación de ejemplares de herbario, sino también en ejemplares vivos en el campo.

\section{Agradecimientos}

Los autores agradecen a la Dra. Marta Morbelli por el permanente apoyo; al Sr. Federico Castía del Ministerio de Ecología de la Provincia de Misiones, por su excelente asistencia y buena predisposición en los viajes de campo y al cuerpo de guardaparques de la Reserva de la Biosfera Yaboty. El presente trabajo fue financiado por los siguientes subsidios: PIP 0169 (CONICET), N709/11 (UNLP).

\section{Bibliografía}

TRESSENS, S. G., H. A. KELLER \& V. REVILLA. 2008. Las plantas vasculares de la reserva de uso múltiple Guaraní, Misiones (Argentina). Bol. Soc. Argent. Bot. 43: 273-293.

LABIAK, P. H., SUNDUE, M., ROUHAN, G., HANKS, J. G., MICKEL, J. T. \& R. C. MORAN. 2014. Phylogeny and historical biogeography of the lastreopsid ferns (Dryopteridaceae). Amer. J. Bot. 101: 1207-1228.

MARQUEZ, G., G. E. GIUDICE \& M. M. PONCE. 2006. Pteridofitas de la Reserva "Valle del Arroyo Cuñá Pirú" (Misiones, Argentina). Darwiniana 44: 108-126.

MORAN, R. C., J. PRADO \& P. H. LABIAK. 2009. Megalastrum (Dryopteridaceae) in Brazil, Paraguay, and Uruguay. Amer. Fern J. 99: 23-39.

MORAN, R. C., PRADO J. \& M. A. SUNDUE. 2014a Megalastrum (Dryopteridaceae) in Andean South America, Part I. Amer. Fern J. 104: 109-178.

MORAN, R. C., PRADO J. \& M. A. SUNDUE. 2014b. Megalastrum (Dryopteridaceae) in Andean South America, Part II. Amer. Fern J. 104: 181-236.

PONCE, M. M. 2001. Additions and corrections to the pteridophyte flora of northeastern Argentina. Amer. Fern J. 91: 70-72.

SEHNEM, A. 1979. Aspidaceae. In: REITZ R. (ed.), Flora Ilustrada Catarinense I (ASPI): 1-357.

SALINO, A \& M. M. PONCE. 2008. Dryopteridaceae. En: ZULOAGA, F. O., O. MORRONE \& M. J. BELGRANO. (eds.), Catálogo de las Plantas Vasculares del Cono Sur, (Argentina, Sur de Brasil, Chile, Paraguay y Uruguay). Monographs in Systematic Botany fom the Missouri Botanical Garden 107: 33-49.

SCHUETTPELZ, E. \& K. M. PRYER. 2007. Fern phylogeny inferred from 400 leptosporangiate species and three plastid genes. Taxon 56: 1037 1050.

SUNDUE, M., ROUHAN, G. \& R. MORAN. 2010. Megalastrum (Dryopteridaceae) of the circumaustral region: Chile, Argentina, and southern islands of the Atlantic, Pacific, and Indian Oceans. Syst. Bot. 35: 461-475.

Recibido el 26 de abril de 2016, aceptado el 30 de junio de 2016. 